\title{
Study of EDIUS Application Skills on the Film Production
}

\author{
Zhu Ding \\ Chongqing College of Electronic Engineering, Chongqing, China,401331 \\ email:632126716@qq.com
}

Keywords: EDIUS, Film Production, Application Skill

\begin{abstract}
The rapid development of computer technology, making non-linear editing technology came into being. Through the non-linear editing software we can make the video editing more convenient and fast, so that the linear editing technology gradually replaced the linear editing technology, video editing plays an important role. Non-linear editing software Edius5.0 with its powerful video editing capabilities, non-linear editing of video clips plays an important role. This article briefly describes the definition and characteristics of the nonlinear editing technology, the author introduces the function of the software Edius5.0, and then follow the "editing material cutting material - special effects editing - audio editing - subtitle creation - video output" , detailing how to use Edius5.0 software for non-linear editing of video.
\end{abstract}

\section{Introduction}

EDIUS nonlinear editing software is designed for broadcast and post-production environment and has a perfect file-based workflow, providing real-time, multi-track, multi-format mixed, synthetic, color keys, subtitles and timeline output, supports all DV, HDV camcorders and video recorders. Based on non-editing software EDIUS6.5 film post-production process, can be divided into material collection, editing and file output.

Open the EDIUS 6.5 editing interface, in the upper left corner of the "file" drop-down menu item selection, select the new project, in the "Project Settings" menu item to set the project file name and location, and then from the default list we need to select the preset. If you want to change the project preset, you can change it by selecting "Project Settings" under the "Design" drop-down menu item, where you can choose from a variety of video options and audio options. When the project is completed, the camera is connected to the computer and can be collected via the "Capture" menu setting. EDIUS 6.5 can be set to capture the way to record, that is, after the first set up, and then encounter the need to collect the signal, select the level of a good collection of items can be set. The signal that collects the video has a lot of flexibility. It can not only a video signal collection of different formats and types of documents, but also in the collection of proxy files generated, which provides a convenient editing.

\section{The Influence of the Application of Nonlinear Editing Technology on the Production of Film and Television Programs}

First, the application of non-linear editing technology in the production of film and television programs can make the display of film and television programs to achieve the best results, so that it not only has the traditional editing technology, but also with the contemporary computer technology to achieve the effective integration of editing results. Through the application of non-linear editing technology, not only make film and television editing management work more convenient, but also broke through the traditional editing technology constraints, the production of film and television programs played a role in promoting.

Second, the application of non-linear editing technology can be part of the film and television 
programs to effectively deal with the video material in a variety of sound to combine, in order to achieve in the film and television programs when the screen and the sound of the effective integration. Therefore, in the film and television sound material integration, be sure to match the film and television screen. Effective audio processing must be based on the process of dealing with audio, based on this, can ensure that the late film and television production in the recording quality.

Third, in the traditional video material editing, it is difficult to complete the editing of its repeated, if many times to edit the film and television material, but also the quality of film and television resources have a certain impact. And non-linear editing technology can be repeated editing video resources will not affect the film and television resources. Non-linear editing technology by film and television works in different places to adjust several times, and then optimize the quality of film and television programs, in addition, but also to a large extent, improve the editing efficiency of film and television editors. Fourth, the application of non-linear editing technology, film and television resources for the clip has laid a solid foundation, can greatly reduce the editor in the film processing error rate. Therefore, the non-linear editing technology for film and television production has a great role in promoting.

\section{Video Material Editing}

Almost all of the editing operations are carried out in the timeline window and the work of the editor in general is the audio, video, still images, subtitles and other material files placed along the timeline and the appropriate editing. To use these materials as you please, you first need to visualize these materials intuitively on the timeline. EDIUS can handle different types of material in the same project and you can edit a variety of formats on a timeline. Editing different sequences is done by switching the sequence buttons above the scale line on the timeline. A sequence is a set of videos, sounds, images, text, etc. that are edited on the timeline. This group of material can be manipulated as a whole. Click the "File" menu to create a new sequence by "New". There are nested functions that can be nested in other sequences, but do not allow themselves to nest. EDIUS6.5 has audio and video track (VA), video track (V), audio track (A), subtitle track (T), right-click track to see a number of settings.

"Synchronize the lock track" is a useful button. When we edit the material, if you need audio and video at the same point in time, or want to edit the good time in front of the additional material then click on this button, it will help us lock the track, keep all V, A, T Track synchronization. The "sync track button" is the master switch that turns on or off the track synchronization. The "track sync lock" button is used to lock a single track.

Cut the most basic way is to move the mouse click on the corresponding icon to operate. First, select the material, Shift + A select the entire timeline material, use Ctrl can be lit one by one to select the material. And then use the "C" key to cut the material that is lit on the track, and use "Shift $+\mathrm{C}$ " to cut the material of all tracks. Use the " $\mathrm{N}$ " key to cut and delete the portion of the current material from the point between the timeline pointer. Use the "M" key to cut and delete the portion of the current clip and the time line pointer.

When you move the clip, notice the Insert / Override Mode button in the upper left corner of the timeline and click on it to toggle between insert modes and overlay mode, which determines whether the clip is inserted in front of the original material or when the clip is placed Material cover. The basic way to move a material is to light the material and drag it with the mouse. When you press "Alt + Shift" at the same time and drag, all the material after the same track will be affected and moved back and forth.

The transition is to partially overlap the two moments of the adjacent time, so that the material between the material to achieve the desired visual effects. EDIUS6.5 gives us a variety of transitions to choose with a mouse drag can be achieved. The transition duration can also be set, place the timeline pointer between the two timeline clips, press "Alt +1 ", create a 1 -second transition, and so on, "Alt +2 " create 2 Seconds transition, "Alt + 3" to create a 3 second transition. Normally, the switch between the video to keep about two of the stack, the effect will be better.

If you want to achieve in the same video acceleration and deceleration in the integrated use to 
create a special picture rhythm, then we must use the time remapping function. The use of time remapping can achieve "stepless speed" function, which is to achieve the video material speed changes smooth and smooth, no pause, which is the ordinary speed tool can not be achieved. We found that the time remapping tool uses keyframes to control the material's playing time. Click the Keyframe Settings button at the top of the dialog box to create a new keyframe. First select it then it turns green, when the mouse moves to the top when there will be a left and right direction of the arrow, for the left and right drag. First drag the key frame to the left, play the timeline, found the first two key frames between the material content to accelerate the playback, the remaining material into a slow. Drag this keyframe from the initial position to the right, and the contents of the first two keyframes become slow play, and the rest of the material becomes accelerated. It can be seen that the distance between the two key frames is greater than its initial distance, this part of the material slow playback; the distance between the two key frames is less than its initial distance, this part of the material quickly play. Through the key line above the red line, you can determine whether the key frame has been adjusted.

Clerhers use different materials, their size does not necessarily meet the requirements, usually EDI-US will automatically zoom, when the material aspect ratio and the project default is not the same, there will be a black edge when the zoom, black Edge of the way is, first of all need to adjust the material dragged into the timeline, light material right click on the "layout" will appear video layout interface. As shown in Figure 1. For example, click on 3D, first in the lower left area of the interface, in the "video layout" and other options in front of the small box in the check box, and then select / add key frames, different timelines in the timeline set different parameters, including the original material of the cut, re-sampling method, the rotation angle. This can be achieved on the video screen in different directions of flying into the fly, zoom rotation and other operations, you can achieve the emphasis, the order of the spread of the same effect, the video screen changes varied, will produce extraordinary visual impact. This video is generally used as film titles or intermediate interspersed fragments.

The clip does not lose picture quality, and the output format of the file is the key to determining the quality of the picture. The principle of selecting the output video file is that the picture quality is clear and the space is small. In the EDUIS6.5 available in the video format AVI, canopus HQ, H.264 / AVC, GF, K2, MPEG, P2, WMV and so on, each format also provides a variety of options The According to experience, the author generally choose MPEG-2 file format, the image size is usually selected 720p, so that you can in the quality of the clarity and space occupied by the size of the two aspects of the optimal balance. Sometimes standard definition video output quality cannot meet the requirements, then we can only on the color and then under the kung fu, such as drop saturation, plus point contrast can also make the screen look clear.

\section{Conclusion}

Non-linear editing has the characteristics of strong networking and high integration, and its computer-based platform is combined with the production of film and television programs. It is the way to store the video resources into the hard disk and the deep innovation of the traditional linear editing. The Non-linear editing not only completed the output of digital video, which for video clips also have a certain role in promoting. In addition, the production of video effects also have a certain role in promoting, and to minimize the program production staff turnover rate, the non-linear editing technology for film and television production to bring the advantages of the ultimate play. EDIUS use of EDIUS process and editing techniques, the material taken for the special effects of the added and reasonable packaging, technology and video editing work to achieve a multiplier effect, while better performance as a film reflects the technology point, especially EDIUS is supported by the multi-file format output, the production of video can be uploaded to a different publicity platform, give full play to the role of science and technology in science and technology propaganda work. 


\section{References}

[1] Du Dan. How to watch high-definition programs at home [J]. Modern Television Technology. 2009 (02)

[2] Wang Dongxue. How to solve the military theater to watch high-definition programming[J]. Modern film technology. 2008 (11)

[3] Wang Guoxing. Construction of the real process can manage the high-definition program production platform [J]. Modern television technology. 2011 (08)

[4] Wang Xiujun. Receiving / playing high-definition program two affordable (medium) - audio and video reception[J]. Satellite TV and broadband multimedia. 2008 (08)

[5] Li Jing. Read the Japanese BS USB receiver box Friio [J]. Satellite TV and Broadband Multimedia. 2010 (08)

[6] Wu Shengquan. Two kinds of high-definition programs under the transformation of the test and analysis[J]. Modern television technology. 2011 (12)

[7] Mei Jianfeng. Talking about the high-definition program technical standards and development significance[J]. China Cable TV. 2009 (09) 